\title{
Healthcare Delivery to the rural area in Iran
}

\author{
Hooshang Lahooti*1, Kevin $\operatorname{Rod}^{2}$ and Alayar Kangarlu ${ }^{3}$ \\ ${ }^{1}$ Department of Medicine, University of Sydney, Australia \\ ${ }^{2}$ University of Toronto, Canada \\ ${ }^{3}$ Department of Psychiatry, Columbia University, USA
}

*Corresponding author: Hooshang Lahooti, Department of Medicine, Thyroid Research Program Sydney Medical School Nepean Campus, University of Sydney, Level 5, South Block | Nepean Hospital| NSW | 2747, Australia.

To Cite This Article: Hooshang Lahooti. Healthcare Delivery to the rural area in Iran . Am J Biomed Sci \& Res. 2019 - 3(5). AJBSR.MS.ID.000709. DOI: 10.34297/AJBSR.2019.03.000709

Received: June 25, 2019 | Published: July 03, 2019

\begin{abstract}
The population explosion in Iran since 1979 uprising, mismanagement, and widespread corruption in the various echelon of Islamic government hierarchy has strained the neglected social infrastructure that has not kept up with the demands and changing demographics. This effect is apparent in the provision and delivery of primary healthcare to rural areas. The contributing factors, such as lack of health education to prepare sufficient numbers of health professionals and the distribution of medical services has not equitably reached all corners of the country. The significant gaps in accessing the medical care between different socioeconomic strata affected the rural healthcare delivery profoundly compared to urban settings. The healthcare system is part of the infrastructure, and its implementation is for the good of all Iranian and not as a business enterprise. Having said that there is a need to develop and implement a comprehensive universal/national healthcare policy; this can be achieved by considering the previous achievement and recognising the importance of solving future complex problems.
\end{abstract}

This healthcare policy should cover the health education, delivery of healthcare and an efficient system free of corruption for reimbursement of healthcare professionals. It is essential to address these problems reasonably well to prevent the occurrence of them in the future secular government in Iran if ignored, that will prevent the recovery and revival of the nation at all levels.

The new system should entail a non-profit government funded a system that is a universal, centralised/single-point healthcare insurance in Iran for all Iranian. The point to emphasise is that delivery of health; dental and pharmaceutical cares are not subject to entrepreneurship and profitmaking facilities. These are the fundamental needs of a society that is provided by a government. The funding for the healthcare is by collaboration with the universities educating the necessary health professional and financial support by pharmaceutical industries, private sectors and the government.

\section{Introduction}

Iran has 31 provinces, an area of $1648195 \mathrm{~km} 2$ and a population of approximately 80 million people, which composed mainly of the young. Iran's population is mostly urban and of note is the people of the urban areas increased by about $70 \%$ due to rural-to-urban migration and the designation of rural centres as towns. In 1975, the Iranian government of the time established the Ministry of Health and Welfare attempting to provide a coordinated approach to Medical education and delivery of healthcare in the country. In 1986, the present Islamic Government established the Ministry of Health and Medical Education to supervise the training of health professional.

The ministry is responsible (A) for improving the quality of health professional education and training and (B) promote the supervisory role of Medical Schools across the nation. The Ministry is the ultimate authority to regulate the licencing and the activities of public and private sector healthcare providers in the country [1].

\section{Framework for Rural Healthcare}

People in rural areas of Iran need comprehensive healthcare plan. The achievement of this vision requires the fulfilment of the following five goals:

\section{Rural regions in Iran will have}

a. Availability of comprehensive Healthcare.

b. Delivery of reasonably well-designed, appropriate and continues healthcare service.

c. The government maintains the Healthcare delivery with skiled, well-trained and well-supported health workforce. 
d. All the sections in the healthcare delivery service should collaborate in planning and policy development.

e. The healthcare delivery service should have, transparency, accountability and strong, decisive leadership and governance.

\section{The Healthcare plan for the rural area should incorporate the following principles}

a. Provision of sustainable healthcare services tailored appropriately around the population needs.

b. The design of appropriate healthcare plan based on geographical and socio-cultural needs of rural residents.

c. Appropriately helps the flexible, realistic, original, safe and high-quality healthcare services.

d. Maintenance of the existing health workforce reasonably well to support future needs.

e. Involving local communities and utilising their abilities and potential in developing the delivery of healthcare according to the needs of the locality.

f. The local community is actively engaging the government, industry, healthcare professional to continue with the appropriate reforms to deliver better healthcare services.

g. Application of the evidence-based knowledge of the locality needs to develop a tailored healthcare delivery appropriately.

h. The planning and delivery of healthcare system should be transparent and accountable.

i. Acceptance and apply new and existing technology.

In Seeking Ways to Improve the Planning and Delivery of Healthcare in Rural Iran

\section{Five areas need consideration and attention, namely}

a. Access

b. Service models and models of care

c. Health workforce

d. The collaboration of the local community and other active partners for planning at the local level

e. Strong leadership, governance, transparency and performance.

Each of the above areas links directly to one of the five key goals

a. Regional centres require establishing specialist services in the locality

b. Healthcare services, aged care, need coordination and integration with community services.

c. Observing, considering the culture of rural residents in providing appropriate care for rural residents. d. Government assisted patients travel scheme.

e. Government providing specialist services; using mobile healthcare facilities to remote and inaccessible rural areas.

f. Government supporting the emergency services, for example, aircraft carrying doctors and auxiliary health personnel for emergency surgeries and supporting the initiative to use information technologies, including telehealth and e-health initiatives.

The Islamic Government Ministry of Health and Medical Education introduced "Family Physician Program" an innovative idea, but regrettably, physicians were not trained for challenges of the rural area and not reaching a vast number of people to provide quality care. To rectify this pitfall, the participating physician encouraged to enrol in a short course at the beginning of their contract, and further learning with an internet-based continuous professional development program. The biggest obstacle to this program was the finances promised by the ministry of welfare and massive budget cut by the department of health.

In Future, the "Mobile Clinics" for rural areas are essential to serving remote areas of the country. Hospitals and clinics belong to the government and operate by the government in cooperation with local communities to provide the necessary healthcare for population both in urban and rural areas. The Health Delivery System to the rural area in Iran should search for the best existing models around the world and improve upon the successful systems already in place in other countries. This healthcare system needs to support the primary existing rural clinics - i.e. Hospitals and other clinics in the cities, and Support Primary Clinics at other less populated areas. Japan healthcare delivery is one of the advanced systems, and it would be appropriate to consider such a method for the future in Iran.

The Organizational structure of Public health system in Iran

a. At the national level

b. At the provincial level

c. At the township and rural level

Ministry of Health and Medical Education exercise its authority at the national level responsible for planning, governance, policymaking, financing and supervising the national health and medical education programs. Universities of Medical Sciences and Health Services are a responsible authority at the provincial level to provide the necessary healthcare tailored for the individual, population-based and environmental affected health issues. A District Health Network, which consists of a district health centre, urban and rural health centres, health posts and health houses, is the responsible body for the township and rural level of healthcare in Iran. The provincial and municipality Welfare Organisation's as well as Insurance companies cover part of the cost of healthcare services. Medical schools provide healthcare free of charge or patients pay a minimal amount [2] (Figure 1). 


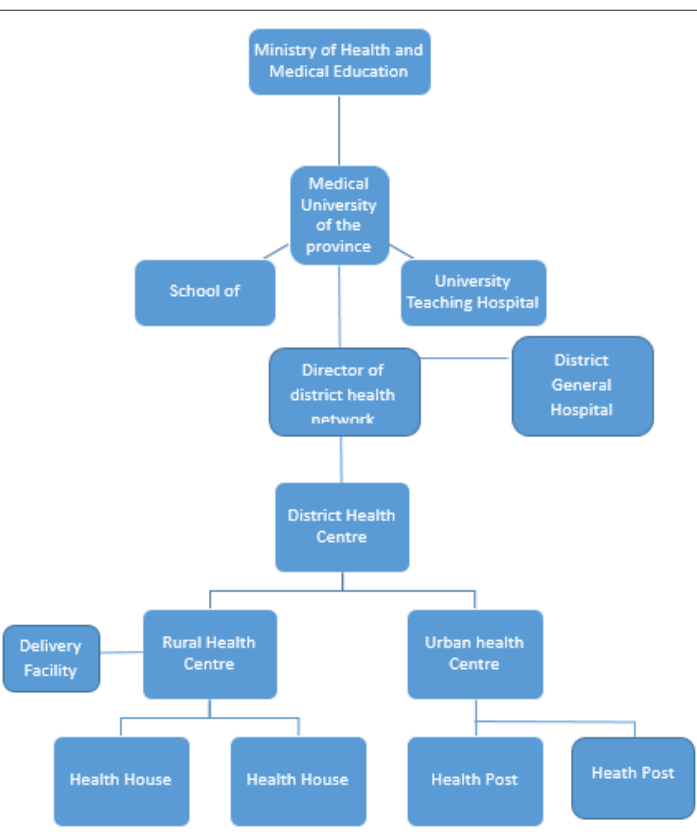

Figure 1: Ministry of health and medical education organisational flow chart.

Patients in rural areas attend the health houses, and the first point of contact with a health professional is "Behvarz" which are male and female, and who serve a population of about 1500 on average. Behvarz has either a primary or secondary education plus two years of training and lives in the locality. Behvarz offers necessary services and plays an essential role in primary healthcare, provide healthcare services such as family planning, case finding and follow up, mother and child care, vaccinations, prenatal and postnatal care, oral health, health education, nutritional suggestions, growth monitoring, treatment for minor illnesses, management of common infectious and chronic conditions, environmental and occupational health services. The personnel in Rural Health Centres are a physician, health technicians and auxiliary nurses, but rarely fully registered nurses. Rural health centres function to prevent the spread of illnesses and provide treatment for patients referred to by the health houses.

The central government cover almost the entire primary healthcare. The Behvarz played significant roles in controlling mortality from the infectious diseases, performing and following up vaccinations, mother and child healthcare, and as a result, the average life expectancy increased substantially. The trends in the epidemiology of diseases have changed from infectious to chronic such as diabetes, cardiovascular and old age illnesses. In 2005, the Islamic Republic government introduced a reform, which covered all villages and towns with populations of up to 20,000 and had two components: The Family Physician Program [3] and the introduction of Rural Insurance Card. In about three years after the opening of the reform, about 6000 physicians and 400 midwives enrolled in primary healthcare. Policymakers assumed that change would reduce hospitalisation. In reality, the reform did not reduce admission to the rural population.

\begin{tabular}{|c|c|c|c|}
\hline Table 1: Health indices in Iran just before the introduction of Primary Health Care (1981), in 2000 and 2011. & $\mathbf{2 0 0 0}$ & $\mathbf{2 0 1 1}$ \\
\hline Health index & $\mathbf{1 9 8 1}$ & 1.24 & 1.3 \\
\hline Population Growth Rate (\%) & 3.9 & 28.6 & 22 \\
\hline Infant Morality rate, death /100 live & 9.4 & 69 & 7 \\
\hline General Life expectancy (years) & 46.7 & $>90$ & $>93$ \\
\hline Overall Vaccinatiom Coverage (\%) & 40 & & \\
\hline
\end{tabular}

The patients living in rural areas did not have ready access to outpatients care such as visits to physicians who were not on the list of primary care, specialist visits, laboratory and radiology services and medicines available at private pharmacies. Outpatients care were not free and located in urban areas. The bureaucracy and lack of proper finance by the government made it challenging for rural residents to access hospital care. The Medical Services Insurance organisation (MSIO) issued free of charge a "Rural Insurance Card" to rural residents. The MISO would cover $90 \%$ of hospital costs only if the rural patients have a referral from primary healthcare service. The rural Insurance card did not include the outpatient's healthcare services, only covered the services provided by the primary healthcare, which were, in essence, already covered by government funding. In 1981, the population growth rate was at $3.9 \%$. However, the introduction of primary health care resulted in a reduction to $1.24 \%$ in the year 2000 and remained stable at $1.3 \%$ in the year 2011. There was a four-fold decrease in the infant mortality rate by the year 2011 [4]. The General life expectancy had a two-fold increase, and overall vaccination increased by two and half fold in the year 2011 (Table 1).

\begin{tabular}{|c|c|c|c|c|}
\hline \multicolumn{5}{|l|}{ Table 2: The demographic characteristics of Rural Health participants. } \\
\hline \multicolumn{5}{|c|}{ Rural Health Care Provider } \\
\hline Profession & Workplace & Level Education & Level of Referral System & Gender \\
\hline Family Physician & Rural Health Centre & General Pactitioner & Second & Male (2) \\
\hline Mid wife & Rural Health Centre & Associate Degree & Second & Female (2) \\
\hline Nurse & Rural Health Centre & Baxhelor Degree & Second & Male (2) Female(2) \\
\hline Community Health worker (Behvarz) & Rural Health Centre & $\begin{array}{c}\text { Secondary School } \\
\text { Diploma }\end{array}$ & First & Male (2) Female (9) \\
\hline
\end{tabular}


Table 2 shows the demographic characteristics of health workers in health centres and health house. It is noteworthy to indicate the role of nurses, which is weak in the Iranian health system and a shortage of trained nurse in rural centres to cover the demand. The nurses mostly considered as a physician help rather than independent caregivers. Despite ongoing changes in Iranian society, the public health system has not evolved according to the population's needs. The challenges facing the rural healthcare system in Iran are provider and client dissatisfaction, limited resources and centralised decision making. The additional problems to the health care system are the lack of attention to primary health care in medical education, the mismatch between the data collection system and new technology. Further challenges are the lack of evidence-based decision making, ignoring the local community in decision making and overlooking the international relation and communication.

The lifestyle, disease pattern and high expectation have changed the characteristics of the Rural Society. The shift from infectious disease to chronic diseases has manifested itself in an increase in the number of diabetes, cardiovascular, hypertension, and psychiatric illnesses- the reason for this change is a lifestyle. The increase in the numbers of patients with hypertension in rural areas is due to low activity and the weight increase (obesity). People in villages used to eat healthy foods, but regrettably, young people have acquired the habit of eating unhealthy fast foods. Residents of the rural areas expect the community health houses medical personnel to dispense drugs for illnesses that do not require medications.

The qualitative studies have identified the complexity of healthcare delivery to the rural areas, which is due to the diversity of the workload of the health houses personnel and the increase in an inability to fulfil the medical demands of the population. Many healthcare issues complicate the rural healthcare delivery such as healthcare providers overloaded with various responsibilities, lack of attendance of rural residents to learn about health education, central government decision making without considering the residents need in rural areas. Increase in the number of populations necessitates the needs for growth in medical care, not enough medical care resources and medications add to the increasing number of the problems facing the healthcare delivery system to the rural areas. In the past, a health worker had to provide family planning healthcare for a small population of 30 but nowadays have to provide for a population of 300 . The inadequacy of training, knowledge and skills of a health provider to face the challenges of chronic disease is an enormous burden on the rural health delivery to rural areas.

\section{Discussion}

The delivery of healthcare to the rural areas of Iran has gone through substantial changes due to the several factors. The shift in lifestyle characteristic has a significant impact on the development of noncommunicable diseases. Before 1979 the health ministry through increase program for prevention of infectious disease by mass immunisation, increase education, and availability of health professional in rural areas managed to control the contagious illness up to $50 \%$ and this trend continued after 1979 by the several reforms introduced by the ministry of health and medical education. The control of infectious disease had reached $90 \%$, which is a credit to the health houses personnel (Behvarz) dedication despite overwork and lack of support from the central government policymakers.

In contrast to the control of the infectious disease, the increase in noncommunicable diseases such as diabetes, cardiovascular, mental health, obesity, and hypertension is on the rise. These are the challenges facing the health houses personnel, which are not adequately trained to answer the rising demand of the rural population. Sadrizadeh [5] also pointed to similar problems which were due to changing pattern of disease, lifestyle, socioeconomic changes, demographic transition by increasing number of the population moving to the urban areas, and nutritional habits. Most infectious diseases are under control, the reduction in mortality because of the infectious disease control is increasing in population due to the longer life expectancy and an increase in the older people, and currently, noncommunicable diseases are the major causes of the mortality. Fully Registered Nurses and Auxiliary Nurses in rural areas cannot answer the ever-increasing demand for better healthcare by rural residents due to the complexity of healthcare that requires them to have specialised knowledge for a broad spectrum of diseases and situations. The problem has become acute because of a shortage of adequately trained, knowledgeable personnel and specialists to treat many of the noncommunicable conditions. The untrained health workers could not provide the required healthcare services required of them, and that results in rural residents losing trust in healthcare offered by the healthcare professionals in health houses and not attending the health house.

The decreasing tendency of rural resident in seeking healthcare have several causes: referral by the health houses Behvarz to the health centres, and low attendance in educational programs. Which are all influenced by sociodemographic, cultural and other factors such as long distance travel from a remote area, and lack of transportation. Shortage of medications in the health house is another factor contributing to the low attendance of rural residents. The major problem in healthcare delivery to the rural areas is the imbalance between the healthcare system and the abilities of the rural healthcare providers to meet the rural community demands. The skills of nurses are underutilised and with adequate training can help and provide limited specialised care that desperately needed in rural areas. This study has a limitation that the findings cannot be generalised due to low sample size. However, the strength of this research is identifying the challenges meeting the healthcare providers in rural areas, and this may have a more wide application to many other rural regions in Iran.

\section{Concluding Remarks}

The rapidly changing rural health care delivery demands the increase in knowledge and training of healthcare provider to meet the high expectation of rural residents' rights for better healthcare. The future agrarian healthcare reforms should take the following recommendation and suggestions: 
a. The control of all the required hospitals and laboratories in Iran which are not- for profit organisation is by a single organisational unit which is composed of the representative of local authorities and the government. The government role is to observe the proper conduct and corruption free functioning of this unit. The funding of healthcare is by the local authorities and the government.

b. All Iranians will enjoy health and medical expenses covered by a national health insurance unit. Iranian will show their national insurance card to the Iranian clinics, hospitals, and health centres. The associated costs of the healthcare services determined by the law and after approval by the local authorities submitted to the government for approval.

c. The renewal of the healthcare professional licence is subject to their participation in the new healthcare system, and the specialist payments charge equal to all other professions specialists in the country. This equal pay to specialists is essential to prevent excessive and unreasonably high costs healthcare specialists' demands from the already impoverished patients that contribute to unnecessary poverty and the increase in the gap between the poor and the rich.

d. The delivery of Health Services to the rural areas in Iran requires Research and Development, which is an integral part of the new healthcare system. It is to use national resources to develop drugs for Iranian patients with the help of government and local pharmaceutical industries. e. An independent commission under the authority of the Parliament is responsible for tariff regulation and monitoring of the quality of services.

f. The government should provide necessary mobile clinics services and experienced professionals to help Iranian in the villages and remote areas of the country.

\section{Acknowledgment}

We are very grateful to His Royal Highness Crown Prince Reza Pahlavi and Crown Princess Yasmin Pahlavi for their continuous support of the Qoqnoos project, and the Directors of the Qoqnoos projects for their immense knowledge and contributing constructive suggestions.

\section{References}

1. Danaei G, Farzadfar F, Kelishadi R, Rashidian A, Rouhani OM, et al. (2019) Iran in transition. Lancet 393(10184): 1984-2005.

2. (WHO) 2006 Health Systems Profile- Islamic Republic of Iran Regional Health Systems Observatory- EMRO, Eastern Mediterranean Region.

3. Amirhossein Takian, Leila Doshmangir, Arash Rashidian (2013) Implementing family physician programme in rural Iran: exploring the role of an existing primary health care network Fam Pract 30: 551-559.

4. Abbas Abbaszadeh, ManijehEskandari, FaribaBorhani (2013) Changing the Care Process: A New Concept in Iranian Rural Health Care. Asian Nursing Research 7(1): 38-43.

5. B Sardizadeh (2004) Primary Health Care Experience in Iran. Medical Journal of Iranian Crescent 7(1): 79-90. 